\title{
Diffusion-Controlled Lengthening Rates of Bainitic Ferrite a Part of the Steel Genome
}

\author{
LINDSAY LEACH, JOHN ÅGREN, LARS HÖGLUND, and ANNIKA BORGENSTAM
}

\begin{abstract}
As a step in the further development of models and databases to support design of new steels, i.e., the "steel genome", the growth of bainitic ferrite plates is accounted for by a thermodynamic and kinetic approach. The thermodynamic aspects are represented by CALPHAD databases and a Gibbs energy barrier for growth $B_{\mathrm{m}}$. Experimental information on ferrite-plate growth rates for a number of $\mathrm{Fe}-\mathrm{C}$ alloys, some of high-purity, are analyzed in terms of a modified Zener-Hillert model and the barrier as well as some kinetic parameters are evaluated. It is found that the barrier varies in a smooth way with carbon content and lengthening rate. In order to improve the agreement with the experimental information it was necessary to adjust the diffusion coefficient of carbon in austenite at low temperatures. It is concluded that the representation of the experimental data is satisfactory.
\end{abstract}

https://doi.org/10.1007/s11661-019-05208-x

(C) The Author(s) 2019

\section{INTRODUCTION}

THE materials genome is the combination of models and databases that supports efficient materials design ${ }^{[1]}$ the aim being to decrease the development time and costs for materials and processes. The CALPHAD type of models and databases for thermochemical and diffusional data may be seen as role models for materials genomic databases. The purpose of the present work is to provide a practical formalism based on the CALPHAD methodology and databases for predicting the lengthening rate of bainitic ferrite, an important part of the bainite transformation. The approach is inspired by the remarkable success of CALPHAD in materials science and engineering.

The bainite transformation in steels is now considered because it has received a lot of attention over the last decade. Such steels hold promises of excellent engineering properties, e.g., high hardness and strength, good wear resistance and toughness, to mention a few. In order to optimize product and performance, models are increasingly being used to facilitate tailoring of material properties.

In this paper we shall not discuss the mechanism of the bainite transformation as such discussions can be found elsewhere, e.g., in References 2 and 3. We will

LINDSAY LEACH, JOHN ÅGREN, LARS HÖGLUND, and ANNIKA BORGENSTAM are with the Unit of Structures, Department of Materials Science and Engineering, KTH Royal Institute of Technology, Brinellvgen 23, 10044, Stockholm, Sweden. Contact e-mail: annbor@kth.se

Manuscript submitted on May 07, 2018.

Article published online April 2, 2019 rather base our approach on the hypothesis that the primary ferritic component in bainite grows by the same diffusional mechanism as Widmanstätten ferrite over the whole temperature range, ${ }^{[4]}$ i.e., the transformation rate is mainly controlled by carbon diffusion in austenite. The approach is developed through a combined thermodynamic and phenomenological model and the parameters in the model will be evaluated from experimental data on starting temperatures and growth rates.

\section{GROWTH OF ACICULAR FERRITE}

\section{A. Modified Zener-Hillert Model}

The present work stems from the classical Zener-Hillert model ${ }^{[5,6]}$ which was recently improved by Leach et al. ${ }^{[7]} \mathrm{A}$ key quantity in the improved model is the thermodynamic barrier for growth, suggested already by Hillert in $1960^{[8]}$ and in the later work by Hillert et al. in $2004 .^{[9]}$ We will simply refer to it as "the barrier for growth", denoted as $B_{m}$ and expressed per mole of metal of formed ferrite, $\alpha$. All molar quantities in the present work are expressed per mole of metal atom, i.e. they are calculated from the normal molar quantities by dividing with $\left(1-x_{C}\right)$ and will be denoted with the subscript $m$. The barrier will be discussed in later sections. The Zener-Hillert equation was based on two major mathematical approximations which were removed in the improved model. The first one is that the capillarity effect was accounted for by the Gibbs-Thomson equation. The second one is the equation for the driving force. Both these equations are direct consequences of the dilute solution approximation which is not needed due to the development of computers and CALPHAD 
methodology. Instead of the Zener-Hillert equation ${ }^{[6]}$ we write for the lengthening rate.

$$
v=\frac{D_{C}^{\gamma}}{2 \rho} \frac{u_{C}^{\gamma / \alpha}-u_{C}^{0}}{u_{C}^{0}-u_{C}^{\alpha / \gamma}}
$$

where $D_{C}^{\gamma}$ is the diffusion coefficient of carbon in austenite and $\rho$ is the radius of curvature of the edge of the growing $\alpha$ plate. The carbon contents are expressed in $u$ fractions and are related to the ordinary mole fraction $x_{C}$ according to $u_{C}=x_{C} /\left(1-x_{C}\right)$. It should be emphasized that $u_{C}^{\gamma / \alpha}$ and $u_{C}^{\alpha / \gamma}$ are the actual carbon contents on the $\gamma$ and $\alpha$ side of the phase interface taking into account the barrier and the capillarity effect for the plate edge with radius of curvature $\rho$ and the $\alpha / \gamma$ interfacial energy $\sigma$. The effect of capillarity thus is contained in Eq. [1] and consequently it looks different compared to the equations in References 6 and 7. $u_{C}^{0}$ is the carbon content in $\gamma$ far away from the growing $\alpha$ tip, i.e., the carbon content of the alloy. The barrier $B_{m}$ and the capillarity effect $V_{m} \sigma / \rho, V_{m}$ being the molar volume per metal atom, require a driving force acting over the interface $\Delta G_{m} \geq 0$. The driving force must be larger than the barrier and the capillarity effect in order for a reaction to occur. Thus, there is a critical temperature above which no reaction is possible. At this temperature $\Delta G_{m}=B_{m}^{0}$ and $\rho \rightarrow \infty$. This critical temperature will be referred to as $W B_{s}$, see Section III.

The driving force depends on the carbon contents on each side of the interface and is calculated from the thermodynamic description of the $\gamma$ and $\alpha$ phase as

$$
\Delta G_{m}\left(u_{C}^{\gamma / \alpha}, u_{C}^{\alpha / \gamma}, T\right)=\Delta \mu_{F e}+u_{C}^{\alpha / \gamma} \Delta \mu_{C}
$$

where $\Delta \mu_{F e}=\mu_{F e}^{\gamma / \alpha}-\mu_{F e}^{\alpha / \gamma}$ and $\Delta \mu_{C}=\mu_{C}^{\gamma / \alpha}-\mu_{C}^{\alpha / \gamma}$. Below $W B_{s}$ the curvature $\rho$ is finite and the driving force $\Delta G_{m}$ is balanced by the capillarity and the barrier, i.e.,

$$
\Delta \mu_{F e}+u_{C}^{\alpha / \gamma} \Delta \mu_{C}=V_{m} \sigma / \rho+B_{m}(v, u, T)
$$

The critical temperature $W B_{s}$ for onset of growth is calculated from

$$
\Delta G_{m}\left(u_{C}^{0}, T\right)=B_{m}^{0}\left(u_{C}^{0}\right)
$$

The growth of carbon-poor $\alpha$ requires carbon transfer over the interface from $\alpha$ to $\gamma$ and thus a drop in carbon chemical potential, i.e., $\mu_{C}^{\gamma / \alpha}-\mu_{C}^{\alpha / \gamma}=\Delta \mu_{C}<0$. The corresponding flux of carbon is expressed as $J_{C}^{t}=-L_{C} \Delta \mu_{C}$. The parameter $L_{C}$ thus represents the carbon cross interface diffusion. It was argued already by Hillert ${ }^{[8]}$ that due to the similarity with the martensitic transformation the mobility of the interface is probably very high and thus we neglect the effect of finite interface mobility. From the steady state condition and a flux balance over the interface one obtains

$$
-\Delta \mu_{C}=\mu_{C}^{\alpha / \gamma}-\mu_{C}^{\gamma / \alpha}=\frac{v}{L_{C}}\left[\frac{u_{C}^{\gamma / \alpha}-u_{C}^{\alpha / \gamma}}{V_{m}}\right]
$$

Equation [5] gives the driving force expressed per mole of carbon transported across the interface. It is expressed per mole of metal atom of the growing $\alpha$ by multiplication with the factor $\left[u_{C}^{\gamma / \alpha}-u_{C}^{\alpha / \gamma}\right]$ and the total barrier then becomes

$$
B_{m}\left(v, u^{\gamma / \alpha}, u^{\alpha / \gamma}, T\right)=B_{m}^{0}+\frac{v}{L_{C}} V_{m}\left[\frac{u_{C}^{\gamma / \alpha}-u_{C}^{\alpha / \gamma}}{V_{m}}\right]^{2}
$$

Inserting Eq. [6] in Reference 3 yields

$$
\Delta \mu_{F e}+u_{C}^{\alpha / \gamma} \Delta \mu_{C}=V_{m} \sigma / \rho+B_{m}^{0}+\frac{v}{L_{C}} V_{m}\left[\frac{u_{C}^{\gamma / \alpha}-u_{C}^{\alpha / \gamma}}{V_{m}}\right]^{2}
$$

The barrier $B_{m}^{0}$ and its dependence of alloy content and temperature will be discussed in the next section. In comparison with the previous treatment by Leach et al. ${ }^{[7]}$ we have added one term on the right-hand side of Eq. [6] which is proportional to the growth rate $v$.

Equations [1], [5], and [7] constitutes three non-linear equations from which the same number of unknowns may be calculated. At a given temperature, alloy composition $u_{C}^{0}$ and a given value of $\rho$ we may thus calculate $u^{\gamma / \alpha}, u_{C}^{\alpha}$ and $v$.

\section{B. Critical Curvature and Maximum Growth Rate}

For a given alloy $u_{C}^{0}$ and temperature $T$ the growth rate may be calculated for any radius of curvature $\rho$. It is then found that the growth rate will be positive only when the radius of curvature is larger than a critical value $\rho_{c r}$ and will increase towards a maximum at some radius of curvature. The critical value is obtained by setting $v=0$ in Eq. [6] and combine it with Eq. [3]

$$
\rho_{c r}=\frac{V_{m} \sigma}{\Delta G_{m}\left(u_{C}^{0}, T\right)-B_{m}^{0}\left(u_{C}^{0}\right)}
$$

In this case $\Delta G_{m}$ is obtained directly by a parallel tangent construction from the composition in austenite equal to the $C$ content of the alloy $u_{C}^{\gamma / \alpha}=u_{C}^{0}$.

In accordance with Zener we shall take this maximum growth rate and the corresponding radius of curvature as the values that should be compared with experiments.

In the classical Zener-Hillert model the maximum growth rate occurs at $2 \rho_{c r}$. However, Leach et al. ${ }^{[7]}$ showed that the growth rate of their modified model has a rather different dependence of the curvature than the original Zener-Hillert model. In fact the maximum occurs at $2 \rho_{c r}$ only at very high temperatures where the dilute solution approximation is valid. In general the maximum could occur at much higher values and has to be calculated for each particular case.

\section{EVALUATING THE BARRIER $B_{m}^{0}$ IN Fe-C}

As mentioned it is observed experimentally that the growth of Widmanstätten and bainitic ferrite occurs below a critical temperature referred to as $W B_{s}$ which depends on the carbon content of the initial austenite, i.e. $u_{C}^{0}$. In the following we shall first consider cases 
where the influence of alloy content on the barrier can be neglected and the treatment will then hold for binary $\mathrm{Fe}-\mathrm{C}$ alloys and approximately for low-alloy steels.

Leach et al. ${ }^{[10]}$ derived an empirical equation for the effect of alloying elements on $W B_{s}$ temperatures from experimental information. They then calculated the driving force $\Delta G_{m}\left(u_{C}^{0}, u_{M}, \ldots, T\right)$ under these conditions using the empirical representation of experimental data and represented it as the function $\Delta G_{m}^{0}\left(u_{C}, u_{M}, \ldots\right)$, where $u_{M}$ is the $\mathrm{u}$ fraction of a substitutional element $M$. Their barrier for onset of growth in binary $\mathrm{Fe}-\mathrm{C}$ is shown as a function of carbon content in mass percent in Figure 1. Their evaluation was based on binary Fe-C alloys. As can be seen it is quite low for low carbon content but increases steadily as the carbon content is larger than ca 0.5 mass percent. The critical driving force is represented by a second order polynomial of content $u_{C}$ i.e.

$$
\Delta G_{m}^{0}=70-4560 u_{C}+178900 u_{C}^{2}
$$

When evaluating $B_{m}^{0}$ from information on the onset of growth the carbon content in Eq. [9] is the carbon content of the alloy, $u_{C}^{0}$. At $W B_{s}$ where $\rho_{c r} \rightarrow \infty$ and $v=0$, Eq. [4] shows that $B_{m}^{0}=\Delta G_{m}^{0}$, i.e.,

$$
B_{m}^{0}=70-4560 u_{C}^{0}+178900\left(u_{C}^{0}\right)^{2}
$$

However, when using Eqs. [6] or [7] it is not self-evident for what carbon content in austenite $B_{m}^{0}$ should be evaluated. We argue that it should be for the carbon content on the $\gamma$ side of the interface because all quantities in Eqs. [6] and [7] are properties of the migrating interface. In general we thus take

$$
B_{m}^{0}=70-4560 u_{C}^{\gamma / \alpha}+178900\left(u_{C}^{\gamma / \alpha}\right)^{2}
$$

The procedure outlined in Section II-B allows the calculation at any temperature $T \leq W B_{S}$ for a given alloy content $u_{C}^{0}$. At each temperature the critical radius of curvature is obtained from Eq. [8] and the growth rate $v$, the contents $u_{C}^{\gamma / \alpha}$ and $u_{C}^{\alpha / \gamma}$ from the solution of Eqs. [1], [5], and [7].

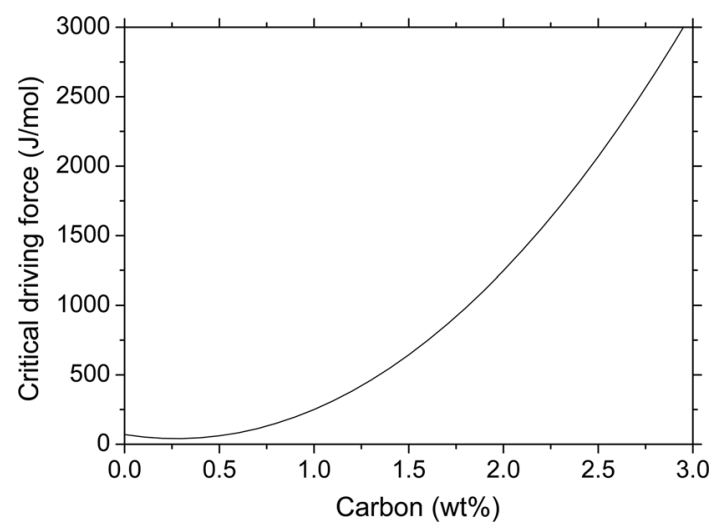

Fig. 1 - Critical driving force $\Delta G_{m}^{0}$ for onset of growth as a function of carbon content in mass percent.

\section{EVALUATING THE UNKNOWN PARAMETERS FROM EXPERIMENTAL GROWTH RATE INFORMATION IN Fe-C}

It is clear that Eq. [4] can represent the onset of growth of bainitic ferrite. We shall now investigate to what extent the present model, see Section II-B, is capable of representing the experimental lengthening rate data. The following quantities are needed to calculate the lengthening rate; the thermodynamic properties of austenite and ferrite, the diffusion coefficient $D_{C}^{\gamma}$ for carbon in austenite, the ferrite/austenite interfacial energy $\sigma$, the barrier $B_{m}^{0}$ and the kinetic transfer coefficient $L_{C}$. The information on thermodynamics available in CALPHAD databases will be accepted and the barrier $B_{m}^{0}$ was discussed in Section III.

\section{A. Diffusion in Austenite}

In principle the diffusion coefficient $D_{C}^{\gamma}$ is available in the CALPHAD databases and it is well established that it increases not only with temperature but also drastically with carbon content. However, in Eq. [1] only a single value enters for a given temperature and alloy composition. This means that some kind of effective value must be used. A simple choice would be the arithmetic mean between the maximum value close to the edge, where the carbon content is highest, and the value far away in the austenite. This average value was chosen in the present work. Nevertheless, it should be kept in mind that the diffusion coefficient in the databases is evaluated from experimental information above the eutectoid temperature where austenite is stable whereas the present application represents a long extrapolation both in temperature and carbon content. Consequently the database value will thus be very uncertain at the lower temperatures where the carbon contents $u_{C}^{\gamma / \alpha}$ becomes very high.

In view of these uncertainties we have chosen to accept the database values of $D_{C}^{\gamma}$ above $1000 \mathrm{~K}$ and take the average value as discussed above. Below $1000 \mathrm{~K}$ we have introduced a correction factor $f_{D}=e^{\frac{Q \text { corr }}{R}(1 / T-1 / 1000)}$ which is unity at $1000 \mathrm{~K}$ and $Q_{\text {corr }}$ is an adjustable parameter.

\section{B. Experimental Data from Literature}

The experimental lengthening rates of bainitic ferrite in binary $\mathrm{Fe}-\mathrm{C}$ alloys were collected from literature as the absence of additional alloy elements allowed for a simpler comparison with the model. These data were therefore used as a basis for the evaluation of the unknown parameters. The microstructures of interest were selected as Widmanstätten ferrite, acicular ferrite, lower bainite, or upper bainite. Ferrite is the leading phase and in principle the formation of carbides is a secondary reaction that occurs after plate lengthening. The presence of cementite in the experimental data used is therefore not thought to have any effect on the lengthening rate unless it formed on the tip or close to the tip of the plate, in which case the diffusion field of 
carbon would be strongly affected. Much of the data fall into one of two categories in terms of the methods used to measure lengthening rates. In situ measurements were done by References 11 and 12 with hot stage metallography. Another method used was to measure the longest plate on a polished plane after isothermal heat treatment from optical micrographs. ${ }^{[8,13,14]}$ For in situ measurements it is not necessarily the longest plate that was observed but rather a typical plate. In certain instances $^{[12]}$ distinct surface and bulk lengthening rates were measured with reference to the spatial location of the acicular plates in the sample. In such instances surface lengthening rates were higher and there was better agreement with the model than with bulk lengthening rates.

We now only use binary $\mathrm{Fe}-\mathrm{C}$ alloys with the carbon contents of the binary alloys are $0.24,0.33,0.43,0.50$, $0.70,0.90,0.96,1.16$, and 1.43 given in mass percent carbon from References 11 through 14. The aim was to be able to predict $\mathrm{C}$-curves for growth rates of bainite.

\section{Optimization Procedure}

The calculation method outlined in Section II was now coded in MATLAB ${ }^{[15]}$ using the Thermo-Calc MATLAB interface ${ }^{[16]}$ and the optimization procedure in MATLAB. The unknown parameters, i.e., $L_{C}, \sigma$ and the correction to the diffusivity were then fitted to the experimental growth rates as discussed in Section IV-B.

\section{Results of Optimization}

The following parameters were found in the optimization:

$$
\begin{aligned}
& f_{D}=\exp [3.53(1000 / T-1)] \\
& \sigma=0.098^{-} \mathrm{Jm}^{-2} \\
& L_{C}=2.34410^{-5} \mathrm{~mol}^{2} \mathrm{~J}^{-1} \mathrm{~m}^{-2} \mathrm{~s}^{-1}
\end{aligned}
$$

where $f_{D}$ is the expression used for the correction factor by which the database value for the diffusivity is multiplied with below $1000 \mathrm{~K}$. At $700 \mathrm{~K}$ it yields a

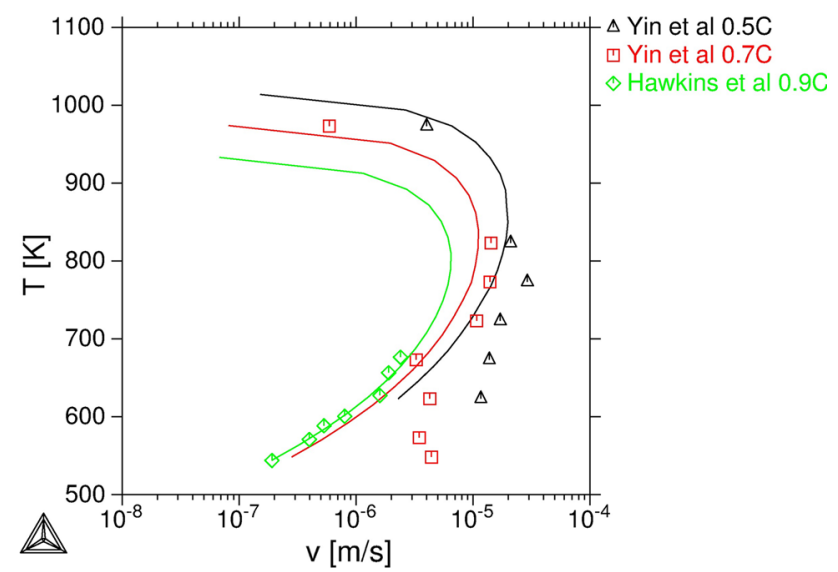

Fig. 2- Growth rates for binary Fe-C alloys used for the optimization. Lines are calculated and symbols are experimental (Color figure online). factor 4.5 and it reduces the activation energy with ca $30 \mathrm{kJmol}^{-1}$.

In Figures 2 and 3 the growth rates for the different binary Fe-C alloys are shown. Solid lines are calculated whereas the symbols are the experimental measurements. The information in Figures 2 and 3 was used in the optimization. Figure 4, with information on a number of low-alloy steels from References 8 and 14, shows data that were not included in the optimization. In Figure 5 we have compared all the experimental information with the simulations. The red squares denote the information used in the optimization.

The fitted diffusivity (dashed line) is compared with the database value calculated for $u_{C}=0.03$ (solid line) in Figure 6. The optimized interfacial energy is somewhat lower than the value $0.23 \mathrm{Jm}^{-2}$ suggested by Hillert. ${ }^{[8]}$

\section{DISCUSSION}

The present approach gives a reasonable representation of the experimental data for binary $\mathrm{Fe}-\mathrm{C}$ alloys. It should be emphasized that in some cases data from different authors are not consistent probably due to experimental uncertainties.

In Figures 2 and 3, calculated growth rate curves are shown with their respective experimental Fe-C growth rates for alloy data used in the optimization. In Figure 2 there is good agreement with the $\mathrm{Fe}-0.5 \mathrm{C}$ and $\mathrm{Fe}-0.7 \mathrm{C}$ alloys though towards lower temperatures, the growth rate data decrease less with temperature. There is a notable disparity in the temperature dependence of growth rates below $630 \mathrm{~K}\left(357^{\circ} \mathrm{C}\right)$ for the $\mathrm{Fe}-0.7 \mathrm{C}$ alloy compared to the alloys with $0.90 \mathrm{C}, 0.96 \mathrm{C}, 1.16 \mathrm{C}$, and $1.43 \mathrm{C}$, which are in agreement with the calculations. The low carbon alloys at high temperatures ${ }^{[13]}$ in Figure 3 also indicate a weaker decrease in growth rate than is predicted by the model. This characteristic could not be reproduced by the present model and in recent work ${ }^{[14]}$ it was tested with composition dependence of diffusivity. The use of a maximum value, $D\left(u_{C}^{\gamma / \alpha}, T\right)$ broadens the curve but the change was not sufficient to capture the effect that would

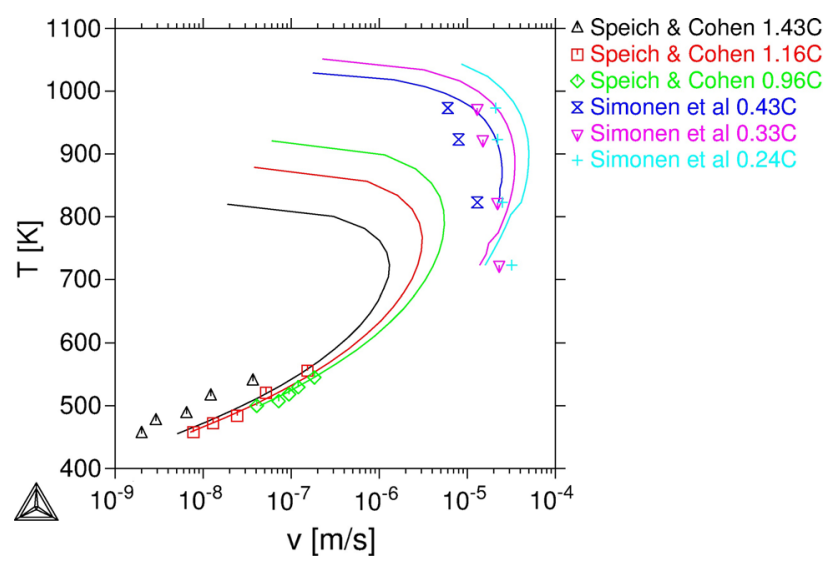

Fig. 3-Growth rates for some binary Fe-C alloys used for the optimization. Lines are calculated and symbols experimental (Color figure online). 


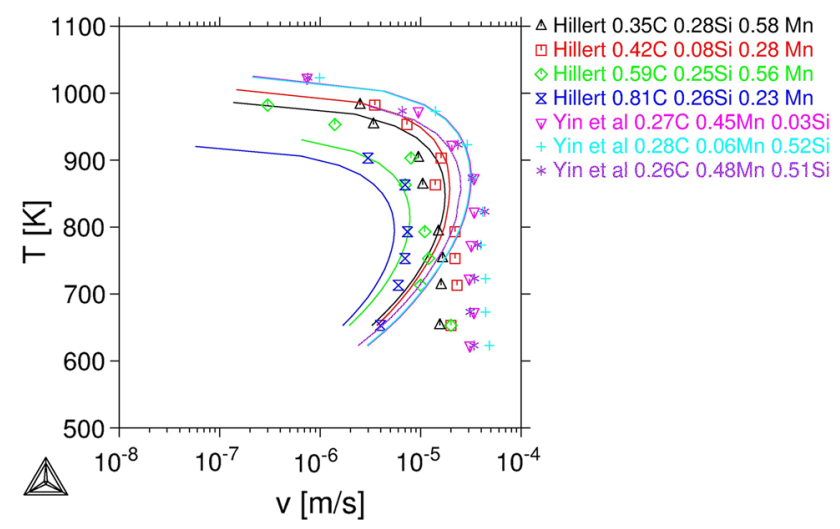

Fig. 4 Growth rates for Fe-C-Mn-Si alloys not used for the optimization. Lines are calculated and symbols are experimental (Color figure online).

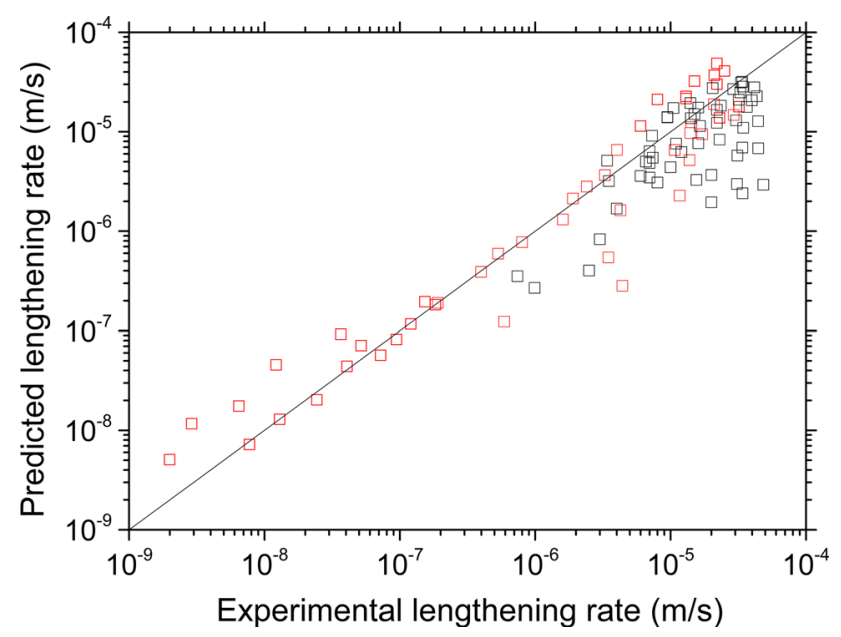

Fig. 5-All experimental lengthening rates compared with the calculations. The ones used are the red squares (Color figure online).

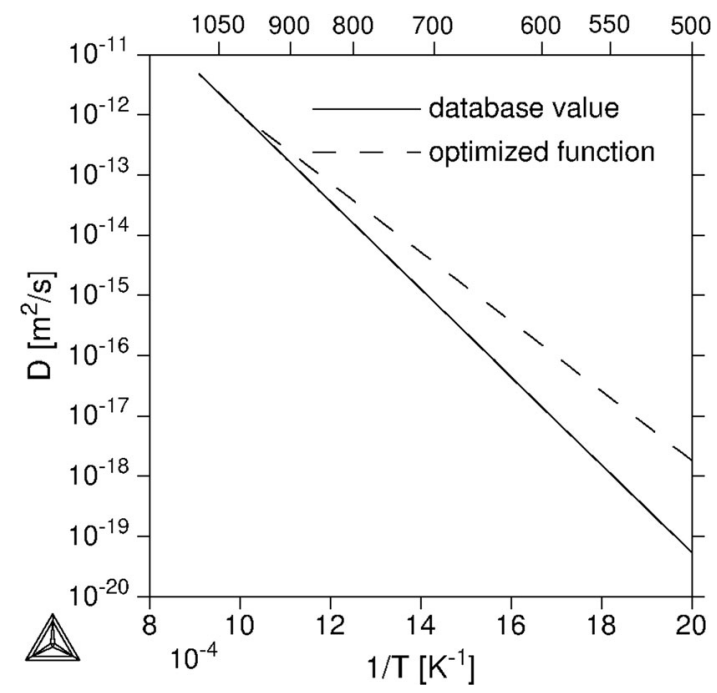

Fig. $6-D_{C}^{\gamma}$, solid line: database value calculated for $u_{C}=0.03$, dashed line: fitted value. cause a flatter curve. It was proposed that such an effect may be related to an increased supersaturation of carbon in the ferrite, in which case some fundamental adjustments would be required to adequately represent the curves. Figure 4 shows comparison between calculated growth rates and experimental data for low alloyed steels not used in the optimization. The high and intermediate temperature data are in fairly good agreement with the calculated lines with the exception of some of the low carbon data ${ }^{[14]}$ at lower temperatures. In spite of this characteristic, the experimental growth rates are satisfactorily represented by the calculated curves. The overall result of all the data examined is compared in Figure 5 and the largest discrepancies correspond to the lower temperature velocity range of the low carbon alloys and of the $0.7 \mathrm{C}$ alloy.

At lower temperatures the model critically depends on long extrapolations of thermodynamic and kinetic properties. We have chosen to rely on established CALPHAD databases for the thermodynamic properties but to adjust the diffusivity. In Figure 6 it is seen that the fitted value of the diffusivity at $500 \mathrm{~K}$ is more than 30 times higher than the database value. The magnitude of velocity can thus be reduced to fit the data by an adaptation of the fitted diffusivity. Evidently, the type of data used bears influence on the optimized parameters and applicability to other types of alloy data. The data used here were binary Fe-C and for the strong ferrite stabilizers $\mathrm{Mo}$ and $\mathrm{Cr}$, the parameters may require due adjustment. Future improvements to the predictive capacity can be made by careful selection of experimental information over a wide temperature range with preference to binary $\mathrm{Fe}-\mathrm{C}$ alloys.

\section{CONCLUSION}

A model for the formation of bainite has been developed on the basis of an energy dissipation approach taking into account existing kinetic and thermodynamic aspects. Important developments are introduced to mitigate the uncertainty of extrapolated diffusivity and interfacial energy. Both parameters are optimized to experimental data and the calculated result is sufficiently satisfactory. Discrepancies observed were in part due to experimental accuracy though the peculiar temperature dependency for low carbon steels would require further investigation in another context. Further work is required to give generic fitting.

\section{ACKNOWLEDGMENTS}

The authors wish to thank Professor em Mats Hillert for valuable discussions. The work was performed within the Competence Centre Hero-m $2 \mathrm{i}$ financed by VINNOVA, the Swedish Governmental Agency for Innovation Systems, Swedish Industry and KTH Royal Institute of Technology. 


\section{OPEN ACCESS}

This article is distributed under the terms of the Creative Commons Attribution 4.0 International License (http://creativecommons.org/licenses/by/4.0/), which permits unrestricted use, distribution, and reproduction in any medium, provided you give appropriate credit to the original author(s) and the source, provide a link to the Creative Commons license, and indicate if changes were made.

\section{REFERENCES}

1. L. Kaufman and J. Ågren: Scr. Mater., 2014, vol. 70, pp. 3-6.

2. M. Hillert: ISIJ Int., 1995, vol. 35, pp. 1134-40.

3. L.C.D. Fielding: Mater. Sci. Tech., 2013, vol. 29 (4), pp. 383-99.

4. A. Borgenstam, M. Hillert, and J. Ågren: Acta Mater., 2009, vol. 57, pp. 3242-52.

5. C. Zener: Trans. AIME, 1946, vol. 167, pp. 550-95.

6. M. Hillert: Jernkont. Ann., 1957, vol. 141, pp. 757-89.
7. L. Leach, M. Hillert, and A. Borgenstam: Metall. Mater. Trans. A, 2015, vol. 47A, pp. 19-25.

8. M. Hillert, Internal report Swedish Institute for Metals Research 1960, reprinted in J. Aggren, Y. Brechet, C. Hutchinson, J. Philibert and G.R. Purdy eds, Les Ulis Cedex 2006, pp. 111-158 (1960).

9. M. Hillert, L. Höglund, and J. Ågren: Metall. Mater. Trans. A, 2004, vol. 35 A, pp. 3693-3700.

10. L. Leach, P. Kolmskog, L. Höglund, M. Hillert, and A. Borgenstam: Metall. Mater. Trans. A, 2018, vol. 49, pp. 4509-20.

11. G. Speich and M. Cohen: Trans. AIME, 1960, vol. 218, pp. 1050-59.

12. M. Hawkins and J. Bedford: J. Iron Steel Inst., 1972, vol. 154, pp. 297-33.

13. E. Simonen, H. Aaronson, and R. Trivedi: Metall. Trans., 1973, vol. 4, pp. 1239-45.

14. J. Yin, L. Leach, M. Hillert, and A. Borgenstam: Metall. Mater. Trans. A, 2017, vol. 48, pp. 3997-4005.

15. The MathWorks, Inc., Natick, Massachusetts, United States. MATLAB 2017b (2017).

16. J.-O. Andersson, T. Helander, L. Höglund, P.-F. Shi, and B. Sundman: CALPHAD, 2002, vol. 26 (2), pp. 273-12.

Publisher's Note Springer Nature remains neutral with regard to jurisdictional claims in published maps and institutional affiliations. 